\title{
Can the comprehensive model phase 4 (CM4) predict the geomagnetic diurnal field for days away from quiet time?
}

\author{
Elvis Onovughe ${ }^{1,2}$ \\ ${ }^{1}$ Department of Earth Sciences, Federal University of Petroleum Resources, Effurun, Nigeria \\ ${ }^{2}$ Jane Herdman Laboratories, University of Liverpool, Liverpool, L69 3GP, UK \\ Correspondence to: Elvis Onovughe (onovughe.elvis@fupre.edu.ng)
}

Received: 21 March 2016 - Revised: 19 July 2016 - Accepted: 22 September 2016 - Published: 18 October 2016

\begin{abstract}
The most recent comprehensive model (CM4) of the geomagnetic field (Sabaka et al., 2004) has been used in conjunction with geomagnetic ground observatory station data to analyse and study the geomagnetic diurnal variation field for days away from quiet time and the CM4 prediction for these times. Even though much has been learnt about many components of the geomagnetic field, the diurnal variation field behaviour for days away from quiet time (moderately disturbed time) has not been intensively studied. Consequently, we analyse these, and the predictive ability of the CM4 for ground variations, and whether the CM4 prediction of the diurnal variation (whether at quiet time or away from quiet time) is valid outside the period of reference that from which the data were used in modelling. In carrying out the study, we compared the observatory station data and the CM4 prediction directly. Using the CM4 code, well-characterised internal and magnetospheric components were subtracted from the data, plots and global maps of the residual field generated and then compared with the CM4 to see how well the model performed in predicting the data at moderately disturbed time $(\mathrm{Kp} \leq 5)$. The results show that the CM4 is valid and produces useful predictions outside the period covering the timespan of the model and during moderately disturbed time, despite the lack of active data in the original model dataset. The model predictability of the data increases as we move to higher spherical harmonic degree truncation, as the model-data misfit is reduced, but with increased roughness as a result of small-scale features incorporated. The observed results show that this relationship between the increase in spherical harmonic degree truncation and reduction in misfit can be restricted by data quality or quantity and global coverage or spread.
\end{abstract}

Keywords. Geomagnetism and paleomagnetism (rapid time variations; time variations diurnal to secular)

\section{Introduction}

Regular changes in the geomagnetic field that take place over a 24-hour period as a result of the Sun's influence on the Earth - the daily or diurnal variation - are one of the most consistent components of the time-varying part of the geomagnetic field (Kane, 1976; Lilley et al., 1999). The daily variations of the geomagnetic field were discovered by English researchers Graham and Watchmaker (1724). The daily variation causes currents to flow in the ionosphere and magnetosphere above us that generate magnetic field signatures (Takeda, 2002), which modify the magnetic field originating deep within the Earth's interior (Langel et al., 1996; Potgieter, 2013; Mandea et al., 2006; Siscoe and Odstrcil, 2008) and locked into rocks of the near sub-surface, the Earth's internal field. Two aspects of these diurnally varying external fields are a large-scale geodynamo, known as Sq, caused by heating on the dayside of the Earth in the ionosphere (Stewart, 1882; Takeda, 2002; ESA SY-001, 2005; Klausner et al., 2013) and the field associated with currents that flows along the geomagnetic equator on the Sun-side of the Earth in the ionosphere, the equatorial electrojet (EEJ) (Kobea et al., 1998; Hamid et al., 2014; Yizengaw et al., 2014). Both of these are reasonably well understood and can be reasonably well characterised in models of the magnetic field when there is little activity on the Sun. However, the solar wind (consisting of charged particles) emanating from the Sun can be modified by activity on or below its surface (Lester et al., 2006; Menvielle and Marchaudon, 2007), such as the emer- 
gence of sunspots, which in extreme cases results in what is known as a geomagnetic storm since it causes changes in the geomagnetic field that can be quite violent. The field changes rapidly over periods as short as minutes and the effects can be detected for up to approximately 2 weeks afterwards as the storm subsides (Gonzalez et al., 1994; O'Brien and McPherron, 2000).

We can use observations of the field to define a number of magnetic indices that indicate the extent to which the field is disturbed, i.e. by how much the ring current is enhanced. Data that are collected when the field is "quiet" according to criteria based on magnetic indices and their rates of change have been used to generate models of the spatial and temporal variability of the various internal and external magnetic field sources (Joselyn, 1989; Hamilton et al., 2015). Relevant indices are used in the temporal parameterization of the external fields. These models are widely used to separate the sources for further analysis, research and application. This includes applying a correction to regional aeromagnetic survey data flown for resource (mineral and hydrocarbon) exploration and it can be necessary to suspend acquisition during geomagnetically disturbed periods since the correction cannot be made reliably. As the field is rarely "quiet", according to our standard definitions (we discard up to $90 \%$ of the data available for modelling, measured at permanent geomagnetic observatories on the Earth's surface and collected by low Earth-orbit satellites; Whaler, 2007), the question then arises as to whether our models are useful outside the strict periods characterised by quiet data.

In this paper, we look at the geomagnetic diurnal variation and the comprehensive model phase 4 (CM4) of the geomagnetic field predictions of ground diurnal variations for days away from quiet time. The paper examines two aspects of the CM4 extrapolation:

1. the extent to which the CM4 describes the diurnal variation during moderately disturbed time, i.e. away from quiet time $(\mathrm{Kp} \leq 5)$, and

2. whether they are valid outside the period covered that from which data were used in modelling.

It is of particular interest to consider the behaviour of the diurnal variation away from quiet time. The CM4 will be compared with ground stations, in particular geomagnetic observatory data. Can satellite models reasonably predict ground variations of the geomagnetic diurnal field away from quiet time, or the data themselves provide real-time constraint? Over the years, much has been learnt about many components of the geomagnetic field, particularly the geomagnetic quiet-time variation (Price, 1969; Stening, 1971; Hibberd, 1985; Campbell, 1997, 1989; Le Sager and Huang, 2002; Takeda, 2002), but the diurnal variation field for days away from quiet time (moderately disturbed time) has not received as much attention as its quiet-time counterpart (Onovughe and Holme, 2015). Using publicly available mea- surements from permanent geomagnetic observatory stations located globally, we analyse the geomagnetic diurnal variation field for days away from quiet time, to study how the field varies and behaves during these times.

\section{The comprehensive models}

The basic idea behind the comprehensive model series is to co-estimate the major field sources using many different datasets. It uses the "comprehensive approach" in a joint inversion of ground-based and satellite field measurements to co-estimate and describe field contributions from core, lithospheric and external (magnetospheric and ionospheric) fields, along with associated Earth-induced signals. The comprehensive models differ from other models in that, as in most other field models, only some of the sources, for example the internal and magnetospheric fields, are modelled separately. Some of these models include the CHAOS series of models (Olsen et al., 2006, 2009, 2010a, 2010b, 2014; Olsen and Mandea, 2008) and the Tsyganenko models (Tsyganenko, 1989, 1995, 1996, 2002). Errors may be introduced into the field model using this approach, as the separation of fields due to various sources may be erroneous. This is because the parameters of the field model at each stage are fitted to a field originating partly from a source not parameterized by the field model.

The comprehensive models were created by Sabaka and Baldwin (1993) and Sabaka et al. (2002, 2004), and based only on quiet-time data from both satellites and observatories. It is not considered predictive nevertheless, for external fields we use it as predictive (Sabaka et al., 2004; Kono, 2007), since the parameterization relies on input of known data such as magnetic indices. Hypothetically, this analysis of ground-based and satellite measurements used together in the comprehensive model allows for the parameterization of all sources, provided the parameters set are treated consistently (Mandea and Purucker, 2005). A separation of the various source fields is facilitated when data from different altitudes are included. The main field (core and crustal) and the induced fields are internal to both satellites and observatories, and the magnetospheric field external to both satellites and observatories, while the ionospheric field is internal to satellites but external to the observatories (Fig. 1). As a result, the use of satellite data can help separate the internal (core and crustal), induced and ionospheric sources from the magnetospheric sources; while the observatory data can help separate the induced (time-varying) fields and secular variation from the fields which are due to ionospheric and magnetospheric fields. Hence, it is theoretically possible to separate ionospheric, magnetospheric, induced, as well as internal fields when jointly analysing observatory and satellite data, if the model parameters are co-estimated.

With the current high availability of satellite and observatory data, global modelling of the geomagnetic field is im- 


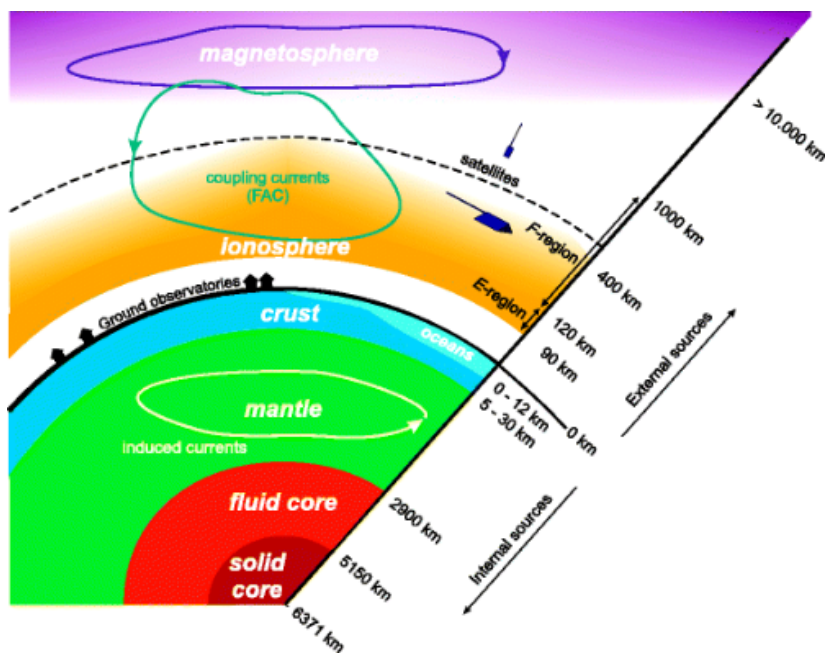

Figure 1. Sketch of the various sources contributing to the nearEarth magnetic field (from Olsen et al., 2010).

proving, providing more useful options when dealing with the removal and separation of various field sources. When considering the external fields (where the diurnal variation field sources originate from), there exist models such as the CM4 (Sabaka et al., 2004) which assist in improved modelling of the geomagnetic diurnal field and provide better understanding of the diurnal variation field. This has come a long way in improving our understanding of the geomagnetic field and the effects produced as a result of its activities.

\section{Geomagnetic data and methodology}

In this section, we first describe the data used to study the geomagnetic diurnal variation field for time away from quiet time. We also discuss the methodology considered in carrying out the study.

\subsection{Data and treatment}

The main interest of the study is to analyse the geomagnetic diurnal field variations for days away from quiet time and the CM4 predictability of such days. To do this, we use hourly mean value series of the XYZ geomagnetic field components from geomagnetic ground observatories. Some of the geomagnetic observatories only have the components available in HDZ. So, we converted the $\mathrm{H}$ and $\mathrm{D}$ components in $\mathrm{HDZ}$ to the $\mathrm{X}$ and $\mathrm{Y}$ in the $\mathrm{XYZ}$ coordinate system respectively, as part of the vector representation of the Earth's magnetic field. As described in Campbell (1989, 1997), we use $X=H \cos (D)$ and $Y=H \sin (D)$, where $X$ is the vertical component in the $\mathrm{XYZ}$ system, $\mathrm{H}$ is the horizontal magnitude and $\mathrm{D}$ is the angular direction of the horizontal component from the geographic north (declination). This system-component conversion does not affect our results as we are interested in

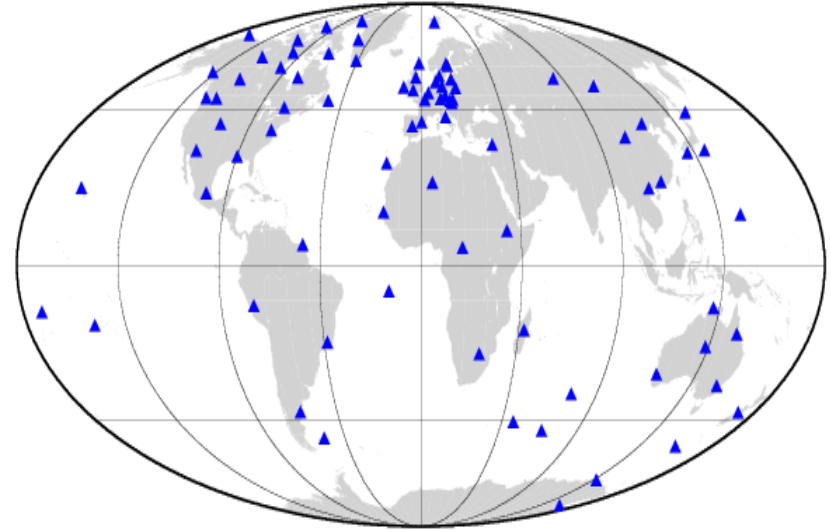

Figure 2. Worldwide INTERMAGNET observatory spatial distribution that provided hourly-mean measurements used in the study.

the geomagnetic variations, and the majority of our observatories are located at low and mid-latitudes. The geographical distribution of the geomagnetic observatories used is shown in Fig. 2. In all, 99 geomagnetic ground observatories in the INTERMAGNET network of observatories provided the data used in this study (http://www.intermagnet.org).

We chose the period of geomagnetic quiet days and moderately disturbed days for this study using the $\mathrm{Kp}$ index to distinguish the quiet days from the moderately disturbed days (Campbell, 1989; Joselyn, 1989). For the quiet days, we use $\mathrm{Kp} \leq 2+$ and for the moderately disturbed days, we use $2<\mathrm{Kp}<5$. The data taken from the 99 observatory locations were scattered all over the globe, as shown in Fig. 2. The distribution is far from uniform, as is inevitable with such studies based on geomagnetic observatory data. The Southern Hemisphere and the oceans are poorly covered compared with the Northern Hemisphere, with its high densities in Europe and North America.

For the quiet days, we only used the data from observatory stations at low and mid-latitudes but we included highlatitude observatory station data for the moderately disturbed days. This is because there are not enough truly quiet days with our Kp selection of $\leq 2+$ in the polar and high-latitude regions due to frequent disturbance events at those latitudes, even during globally quiet days. Above $60^{\circ}$, the magnetic measurements may be completely dominated by magnetospheric processes which impede Sq observation (Campbell, 1989). The study takes into consideration the well-known fact about diurnal variation (particularly the $\mathrm{Sq}$ field) as a largely local time field that can be roughly represented by a current fixed relative to the Sun (Price, 1969). Hence, we use the data provided at universal time (UT) to observe the global variation of the diurnal variation field for days away from quiet time. Adequate knowledge and understanding of the diurnal variation field, particularly away from quiet time, and its associated effects, can only come from considerable and 
detailed analysis of the mean-hourly values of the geomagnetic elements at many observatory locations (Price, 1969).

\subsection{Methodology}

The basic methodology used in this study is based on spherical harmonic modelling of geomagnetic observatory data. The three components of the geomagnetic elements, $\mathrm{XYZ}$, were compiled for each geomagnetic observatory station measurement. Using the "comprehensive approach" in which the major field sources are parameterized and then co-estimated in order to achieve optimal separation of the different field sources (Sabaka et al., 2002, 2004), wellcharacterised internal and magnetospheric field components were subtracted from the geomagnetic observatory measurements. This was achieved by using the CM4 since our major interest is in the geomagnetic diurnal variations, which primarily originate from the external field sources.

Although the CM4 is a quiet-time model, we chose it for the purpose of this study because it is a model which models all the different sources of the near-Earth magnetic field. It is the best and most effective in the co-estimation and parameterization of all the field contributions (from core and lithosphere, and external to their associated Earth-induced signals) (Sabaka and Olsen, 2003). For this study, the CM4 enables us to generate the primary and induced components of the external fields (ionospheric and magnetospheric), excluding a large portion of the field due to the Earth's core. Using the CM4 model, the XYZ components of the field are isolated, so these variations can be studied and compared at different times with the observatory station measurement. This was done first for quiet-time measurements and then for days away from quiet time. To be able to achieve the operation for days away from quiet time, we extended the original lifespan of the CM4 (originally from 1960-2002.5 to 1960-2012). We know that the CM4 was made to primarily analyse the internal field variations of long-wavelength timespan. In its simplest form, the CM4 does the modelling through a threeroute process - input, filter and output. The inputs are the time, position and magnetic indices (Dst and F10.7), which are the driving inputs for the external field terms (magnetosphere and ionosphere) in the spherical harmonic expansion. Dst in particular allows the CM4 to respond to active conditions outside of the original geomagnetic activity remit. As a result, the extension we applied to the CM4 updated the magnetic indices (Dst and F10.7) and the drivers of the external field part of the CM4 code with more recent data (post \pm 2002.5 ) to enable the model to respond to geomagnetic observatory data (away from quiet time) outside the original lifespan of the model.

To further show how the CM4 is performing in globally predicting the geomagnetic diurnal field variations for days away from quiet time, global maps of the residual diurnal variation field are generated from the available geomagnetic observatory station measurements using the extended CM4 at different spherical harmonic degrees and time of day. The extension applied to the CM4 is as explained in Sect. 3.2. Using relative root mean square (rms) differences we also show, quantitatively, the goodness of the CM4 predictions versus the observatory data. With this we are able to analyse the CM4 prediction of the observatory measurement within and outside its timespan.

\section{Results and discussion}

Since we extended the CM4 lifespan to more recent times (post-2002.5) by updating the magnetic indices data (Dst and F10.7), which are the driving force for the external field parts, we needed to test the operation of the model. This was first done for the quiet-time days, since the model is a quiet-time model, before testing its performance for days away from quiet time, i.e. moderately disturbed days, which is the primary interest of this study. The results presented in this study are all diurnal variation conditions, with the simulations performed using the CM4 code for 24 evenly spaced universal time hours. They also provide local variation at specific geomagnetic observatory locations. The observatory station measurements for all the field elements, XYZ, used in generating the plots shown were all centred, i.e. with a mean of zero.

\subsection{CM4 and observatory data comparison}

During quiet time: Fig. 3 shows plots of the comparison of the CM4 with the three magnetic field components, XYZ, of the observatory station data for quiet time outside the lifespan of the CM4 (post-2002.5) for different regions of the globe. The Sq field has been shown to vary smoothly with location and UT (Courtillot and Le Mouel, 1988) as it is a local time field roughly represented by a current system fixed with respect to the Sun. It is also longitudinally dependent (Matsushita and Maeda, 1965). All these features can be seen in the various plots in Fig. 3. The different variation in our plots so reflects the representative or typical pattern at different geographical locations, and these differences observed between all the regions mainly consist of slight phase and magnitude differences (Hitchman et al., 1998; Takeda, 2002).

As expected, the fit between the CM4 and the observatory station data seems to be roughly dependent upon latitude, proximity (i.e. closeness of station locations) and geographical location. As evident in all three magnetic components, $\mathrm{XYZ}$, stations behave in similar ways and show common features, including short-term features recorded simultaneously at all the stations - showing geographical relationship. Generally, the plots show features for the different geographical regions which are in agreement with the Sq variation for the different locations (Campbell, 1982; Chen et al., 2007). While components generated by the CM4 are sometimes not in agreement with those of the observatory station compo- 

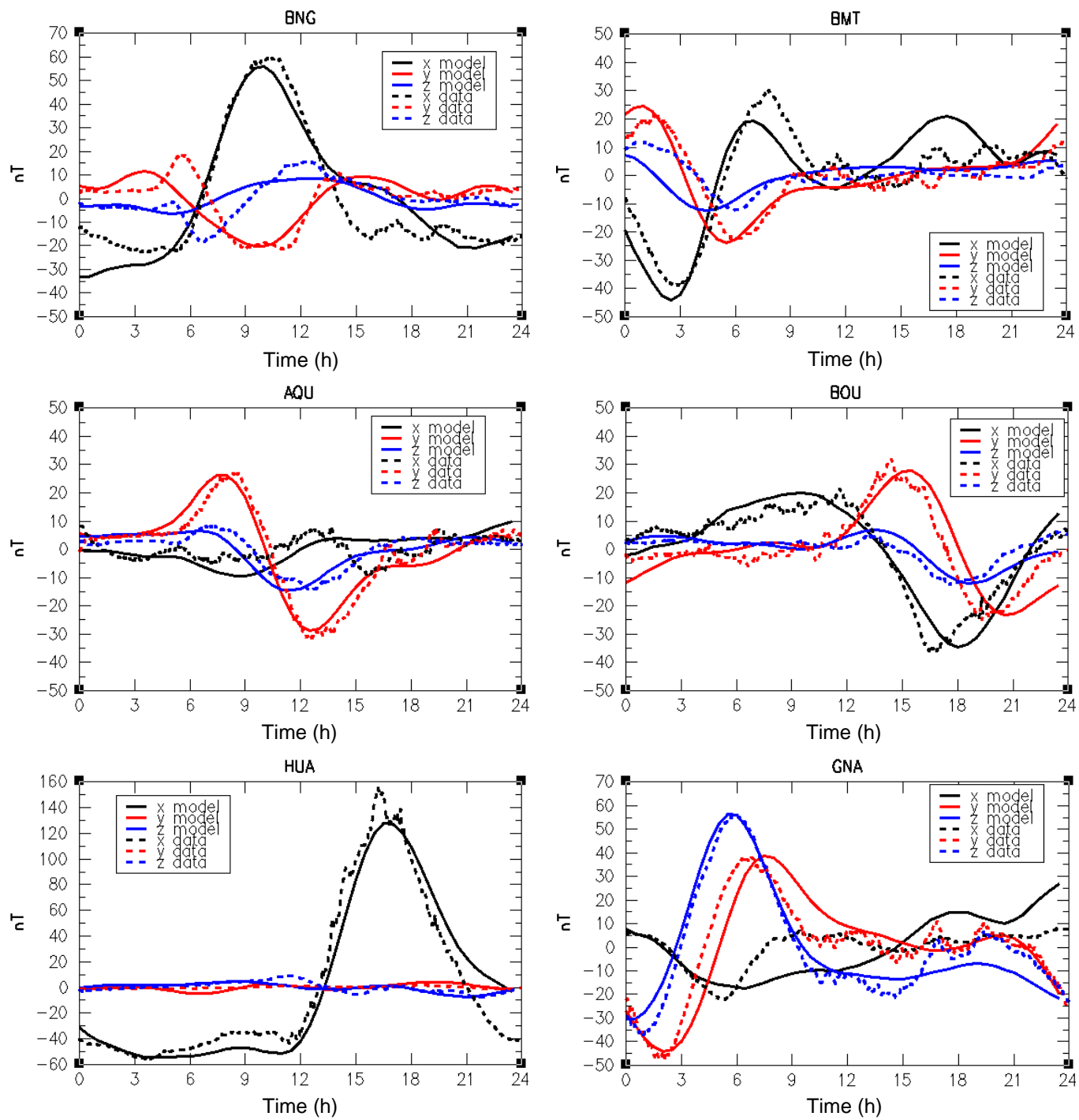

Figure 3. Quiet-time diurnal variation field comparison of the $x$ (black), $y$ (red), $z$ (blue) components of the observatory station data (dashed line) and the CM4 (solid line). These are exemplar plots representative of the different geographical locations of the globe - BNG (Africa), BMT (Asia), AQU (Europe), BOU (North America), HUA (South America) and GNA (Oceania).

nents, they largely show similar patterns, which is encouraging. This indicates that the extension we applied to the original lifespan of the CM4 seems to work.

Away from quiet time: Fig. 4 shows the plots obtained for the comparison between field component data from the geomagnetic ground observatory and the CM4 predictions for the same stations as used for quiet-time period in Fig. 3. As explained earlier, the dataset used to calculate the CM4 is chosen for its lack of geomagnetic activity. For the external field terms, the Dst allows the CM4 to respond to active conditions outside of the original geomagnetic activity remit (Onovughe and Holme, 2015). The plots of the comparison between the components of the geomagnetic observatory station data and the CM4 predictions show Sq variation just as in the case for quiet time. But these are variations that are expectedly more rapid due to higher magnetic-activity period, with fluctuations seen particularly in the $\mathrm{X}$ component. The plots show spatial dependence in the variation, with the components of the observatory station data behaving similarly in different geographical locations, as is to be expected, conforming largely to the observation seen in our quiet-time plots. This includes showing features in common with each other, including the short period variations, showing a kind of global spread. Just as seen in our quiet-time plots, while the CM4 predictions of the geomagnetic observatory data components are not a perfect fit, they show many similar features, with the CM4 seen to produce a better response in predicting the observatory station data than expected. 

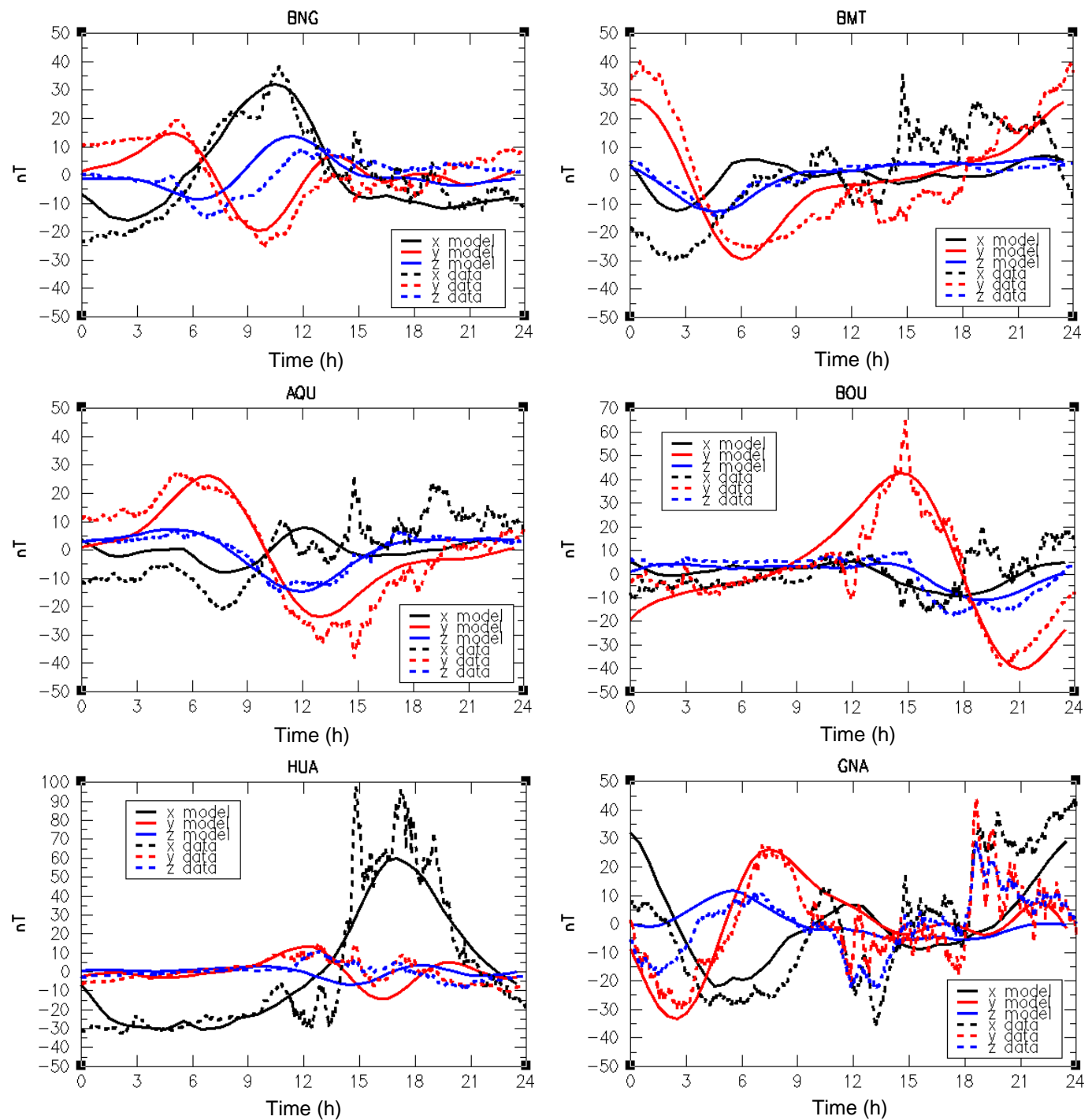

Figure 4. Same as in Fig. 3 but for moderately disturbed time (away from quiet time).

Table 1. Relative root mean square (rms) misfit between the CM4 and observatory data for quiet period (within and outside CM4 timespan) and for moderately disturbed period for each field component $(x, y, z)$.

\begin{tabular}{|c|c|c|c|c|c|c|c|c|c|}
\hline \multirow{3}{*}{ Regions } & \multicolumn{6}{|c|}{ CM4 quiet period } & \multirow{2}{*}{\multicolumn{3}{|c|}{$\begin{array}{l}\text { CM4 moderately } \\
\text { disturbed period }\end{array}$}} \\
\hline & \multicolumn{3}{|c|}{ Within timespan } & \multicolumn{3}{|c|}{ Outside timespan } & & & \\
\hline & $x$ & $y$ & $z$ & $x$ & $y$ & $z$ & $x$ & $y$ & $z$ \\
\hline Africa & 0.51 & 0.44 & 0.77 & 0.55 & 0.51 & 0.78 & 0.90 & 0.83 & 0.93 \\
\hline Asia & 0.65 & 0.50 & 0.75 & 0.66 & 0.51 & 0.78 & 0.94 & 0.87 & 0.91 \\
\hline Europe & 0.38 & 0.32 & 0.51 & 0.40 & 0.38 & 0.55 & 0.69 & 0.61 & 0.72 \\
\hline North America & 0.47 & 0.44 & 0.62 & 0.50 & 0.51 & 0.58 & 0.79 & 0.72 & 0.80 \\
\hline South America & 0.61 & 0.54 & 0.74 & 0.66 & 0.60 & 0.76 & 0.86 & 0.84 & 0.93 \\
\hline Oceania & 0.75 & 0.69 & 0.92 & 0.79 & 0.75 & 0.93 & 0.90 & 0.85 & 0.99 \\
\hline
\end{tabular}


Our observations show that the CM4 produced a better performance in predicting the observatory station data in $\mathrm{Eu}-$ ropean observatories than elsewhere. This may be due to the high density of observatory stations in Europe compared to other locations. This better prediction is noticeable in the $\mathrm{Y}$ component (Fig. 4), and it extends to stations in North America, and to some extent in African and Asian stations. In general, the CM4 performed reasonably well in predicting the components of the observatory station data for days away from quiet time, producing a better prediction than expected. The CM4 matches the regional type features of the observatory station data reasonably well for all three components of the geomagnetic field but not the short-time-period features where we observe rapid variations. This is often where we see the largest misfit between the CM4 and the components of the observatory station data. This is not so surprising due to the limited time resolution of the Dst. However, we can still observe some coherence among the different plots, encouragingly. As primarily a model for analysing internal field variations of the long-wavelength timespan, the CM4's predictive ability of the external field variations beyond the effective range of Dst (i.e. the range of Dst in the data originally used to create the CM4) is an unexpected advantage. Its ability to produce an approximation of the external field, an active time field, variation shows its resourcefulness.

\subsection{Misfit between the CM4 predictions and the observatory station data}

Here we calculated the relative rms misfit between the CM4 predictions and the geomagnetic observatory station data components for quiet time (both within and outside the CM4 timespan) and for the period away from quiet time. This is to enable us to study the consistency of the CM4 in predicting the ground-observatory-station data within and outside the CM4 timespan.

In order to make two different kinds of data comparable, one often looks at the relative rms misfit between the model and input data in field modelling (Maus et al., 2006; Thomson and Lesur, 2007; Hamilton, 2009; Hamilton et al., 2015). For the purpose of this study, we define the relative rms misfit between the CM4 predictions and the observatory station component data after Hamilton (2009) as the following:

$\frac{\operatorname{rms}(\text { data }- \text { model })}{\operatorname{rms}(\text { data })}$

$=0 \rightarrow$ perfect fit (model predicts the data perfectly)

$=1 \rightarrow$ non-correlation (model uncorrelated with the data, i.e. model explains precisely none of the data).

Increasing values away from zero shows an increasingly poorer representation of the data by the model.

Table 1 shows the relative rms misfit values between the CM4 predictions and the observatory station component data. The relative rms misfit was calculated at each obser- vatory location and the average for each region computed with the overall mean relative rms misfit value for each observatory station component from every region of the globe shown.

Since the observatories within each region differ depending on whether the selection is made with the CM4 validity period or outside of it, direct comparison between these two periods for the CM4 and the observatory station data rms misfits is not straightforward, but it is worth commenting on some patterns observed in the relative rms misfit values. The table shows that the mean relative rms misfit values for all the different regions have values less than one $(<1)$. This shows that while the CM4 does not give a perfect prediction of the observatory station data, it shows some fair representation of the observatory component data. Also, the difference between the mean relative rms misfit values for quiet-time period within and outside the CM4 timespan is very small, and even similar in some cases. This justifies the extension of the CM4 timespan outside the period the original data covers. While the mean relative rms misfit value is slightly larger for period away from quiet time, i.e. moderately disturbed period, it still shows the CM4 fairly predicting or fitting the observatory station data, albeit poorly, in some cases. These misfit values are mean values as stated above for observatories at each continent studied. Our observation showed that most individual observatories give smaller misfit values (not shown), but few, especially those close to coastal areas, give higher misfit values, resulting in the mean misfit values to be higher, particularly for the moderately disturbed days. While the misfit values are not a perfect fit, they give a fair representation of the qualitative prediction as shown in Figs. 3 and 4. The CM4 does not give equal predictions in all areas of the globe due to some 20 factors. Our results show that the misfit values are better for $\mathrm{Y}$ component than for $\mathrm{X}$ and $\mathrm{Z}$ components. For $\mathrm{X}$ component, it may be due to the impact of remaining unmodelled contributions and the fact that the $\mathrm{X}$ component is more affected by the external field variations than the $\mathrm{Y}$ and $\mathrm{Z}$ components.

Just as we saw in the qualitative comparison (Figs. 3 and 4), the CM4 predictability as seen in the misfit values is better in Europe than in most other regions. This may be due to a high density of geomagnetic observatories and therefore better quality geomagnetic data in Europe. While the majority of individual stations (not shown) give good misfit results (less than 0.60), the mean misfit for each continent gives much higher values, close to and around 0.90 for the moderately disturbed data.

\subsection{Comparison of global maps of the diurnal variation field}

We investigated further some of the coherence we observed in the plots of the CM4 and the observatory data comparison in Fig. 4. This is done using the CM4 code to create global model maps of the geomagnetic diurnal field at the Earth's 
CM4

00 UT

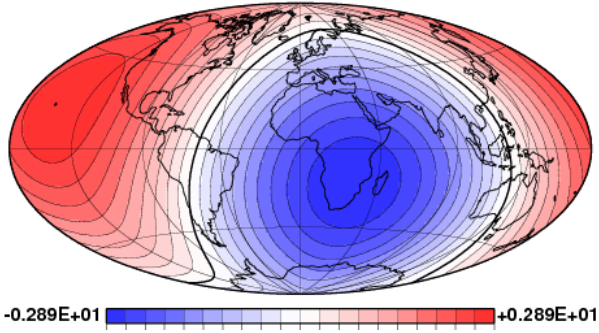

06 UT

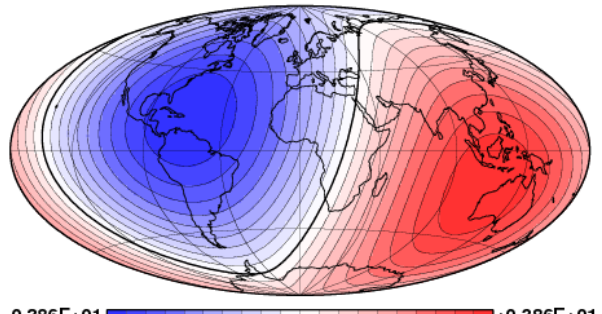

$-0.386 \mathrm{E}+01 \square+0.386 \mathrm{E}+01$

12 UT

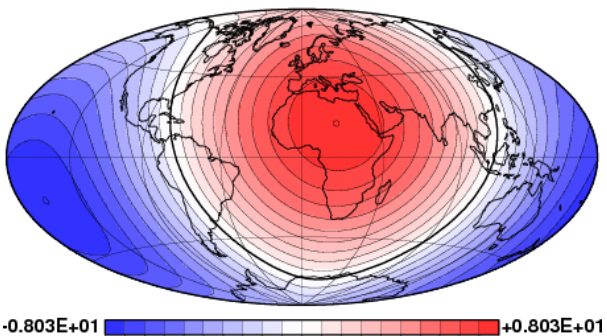

18 UT

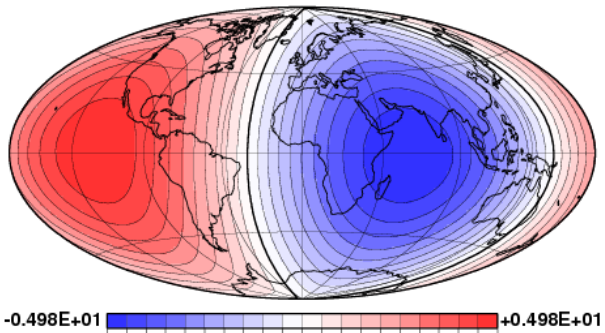

OBS DATA

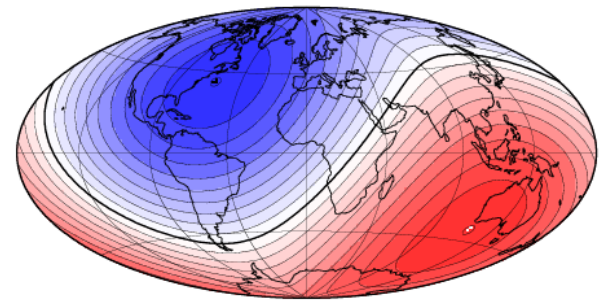

$-0.424 \mathrm{E}+01$
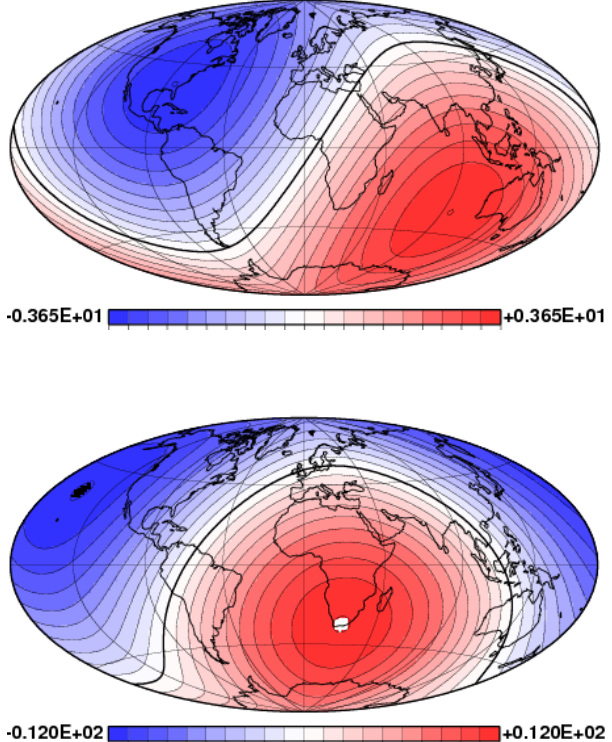

$-0.120 \mathrm{E}+02 \square+0.120 \mathrm{E}+02$

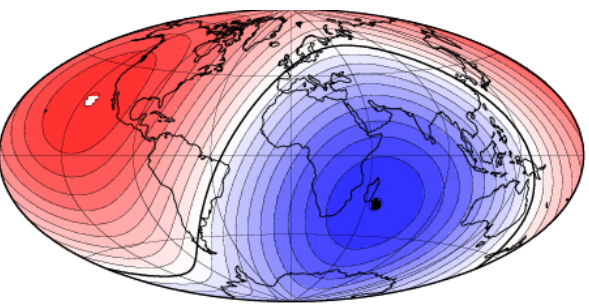

$-0.134 \mathrm{E}+02 \square+0.134 \mathrm{E}+02$

Figure 5. CM4 observatory data comparison of the Z component of the geomagnetic diurnal variation field measured at the Earth's surface at moderately disturbed time, at spherical harmonic degree truncation $n=1$. Left-hand panels are models of data synthesised from the CM4 while right-hand panels are the observatory station data. Note much can be deduced from the map at $n=1$.

surface for days away from quiet time, for the same dates and times as in the plots in Fig. 4. This is done to enable the visualisation and comparison of the CM4 to the observatory station data, to see how well the CM4 predicts the observatory station data.

Figures 5-8 show the $\mathrm{Z}$ component of the diurnal variation field maps at the Earth's surface truncated at spherical harmonic degrees $n=1,3,5$ and 7 at $=00: 00,06: 00,12: 00$ and 18:00 UT respectively. The diurnal variation field maps (left panels) are regularised inversions of the CM4 predictions, and they are also a series of inversions for different maximum spherical harmonic degrees, as stated above. Figures 5-8 show that there is a relationship between the spherical harmonic degrees and the model-data misfit. At spher- 


\section{CM4}

00 UT

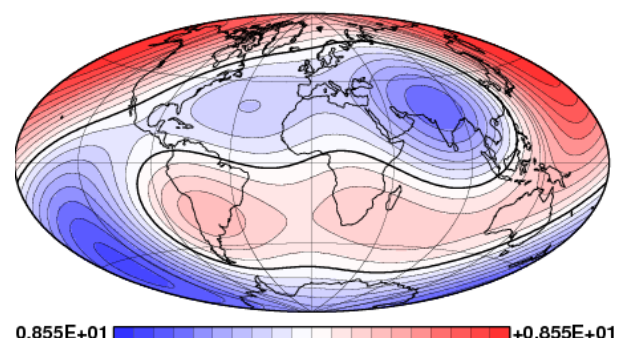

06 UT

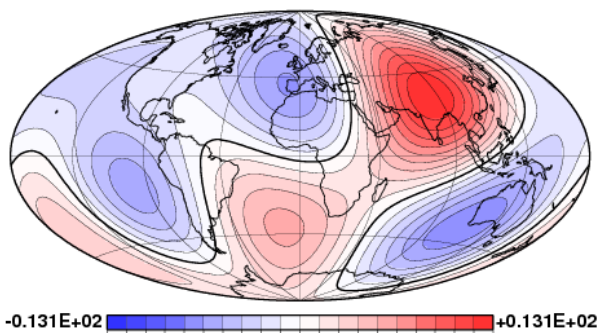

12 UT

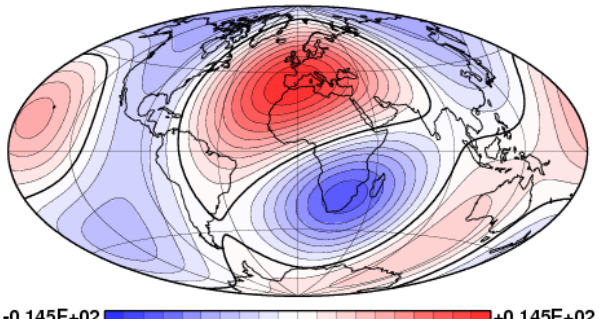

18 UT

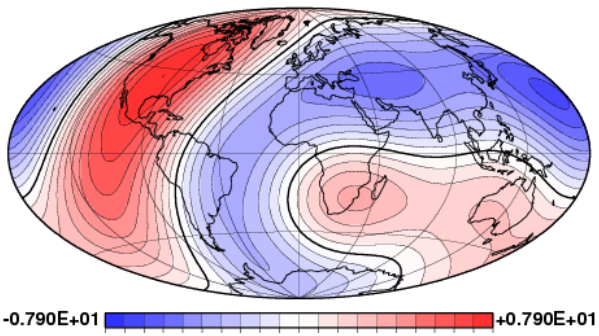

OBS DATA
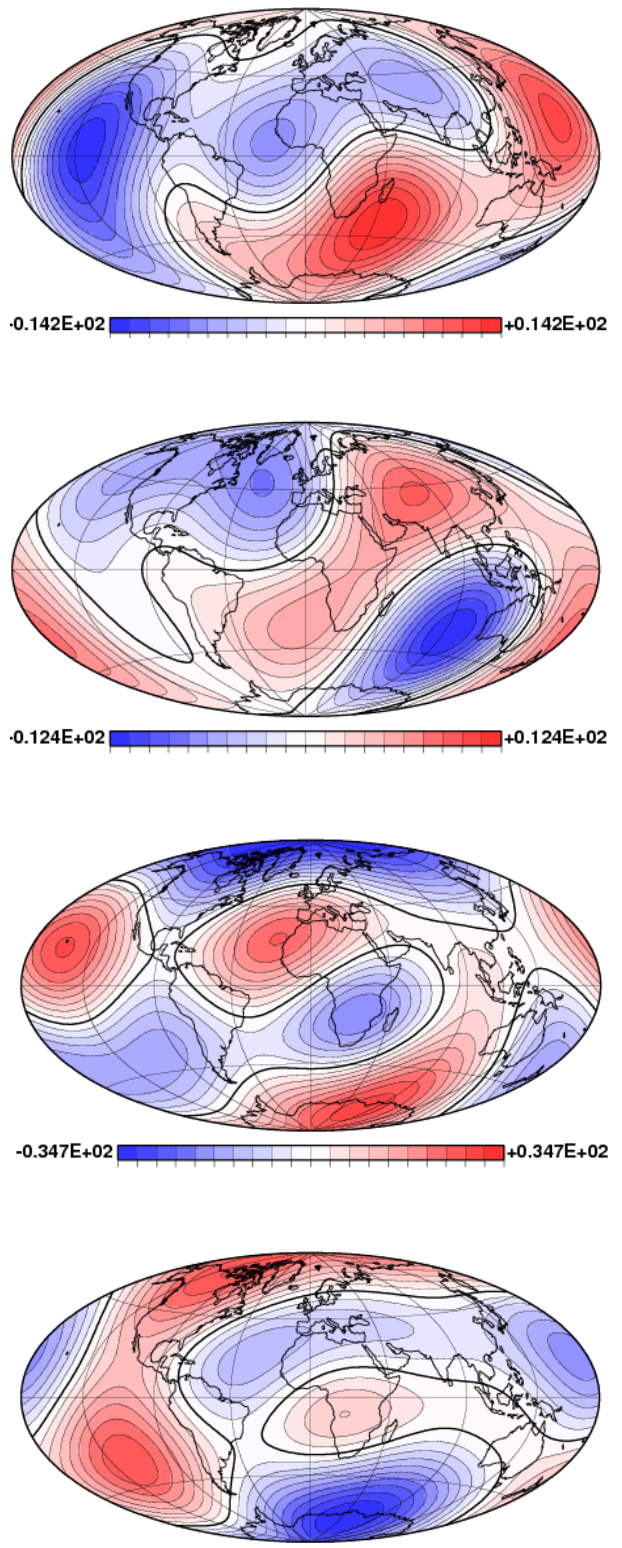

$- 0 . 2 9 6 \mathrm { E } + 0 2 \longdiv { \square } + 0 . 2 9 6 \mathrm { E } + 0 2$

Figure 6. Similar to Fig. 5 but for spherical harmonic truncation up to $3, n=3$. More features start to be revealed.

ical harmonic degree $n=1$ (Fig. 5), the diurnal variation maps basically showed a dipolar structure divided into areas of strong (red) and weak (blue) intensity lobes separated by areas of zero intensity (white). The surface field can only be described by a limited number of large-scale Gauss coefficients. The lobes in the figure appear to be moving from right to left as the day progresses, with the model appearing to predict the data slightly. We can observe some similarities between the CM4 and the data at 06:00, 12:00 and 18:00 UT.
As the spherical harmonic degree truncation is increased to $3(n=3)$ (Fig. 6), more details of the diurnal field are revealed in terms of the areas of strong and weak intensities. Here we can observe some similarities between the CM4 and data at all hours shown. As we increase the spherical harmonic to even higher degree truncation, from 3 to $5(n=3-5)$, more complex details of the diurnal field are revealed (Fig. 7), and as we move to even higher spherical harmonic degree truncation, $n=7$ (Fig. 8), we can see the diurnal field's increasingly complex structure with 


\section{CM4}

\section{UT}

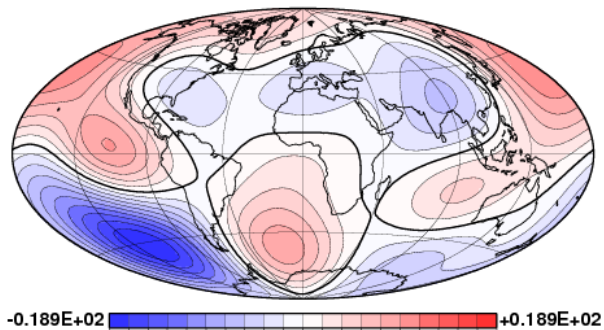

06 UT

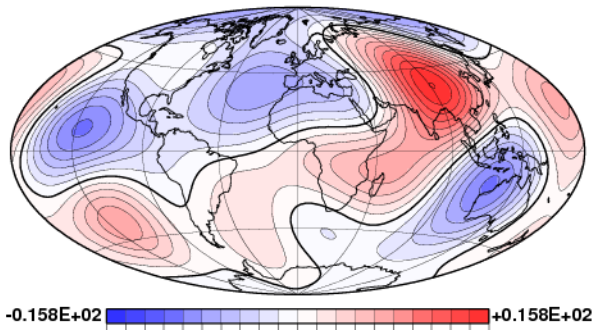

12 UT

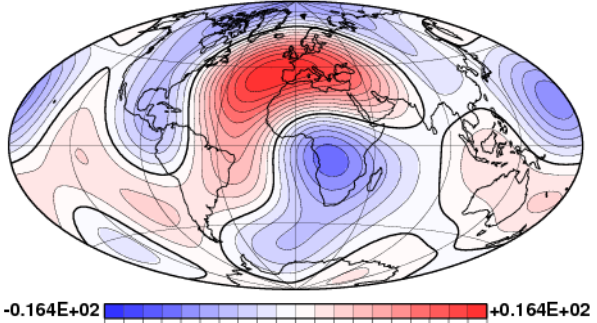

18 UT

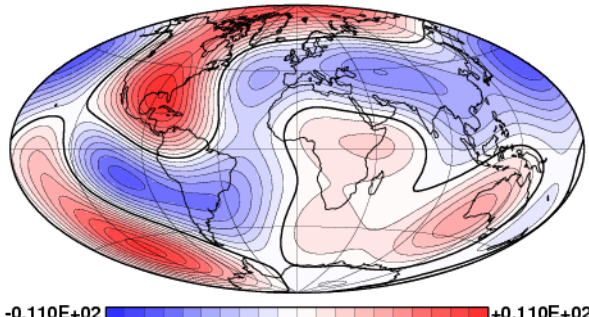

OBS DATA
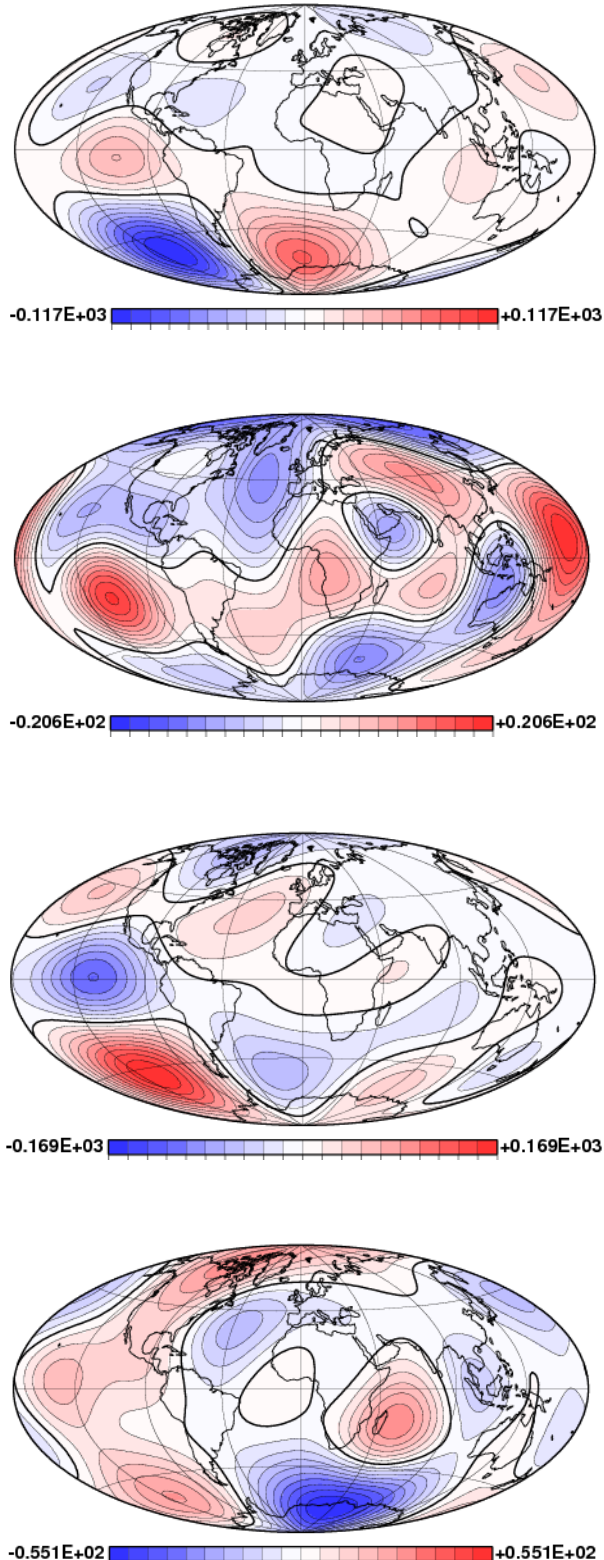

Figure 7. Similar to Fig. 5 but for spherical harmonic degree truncation up to $5, n=5$. More small-scale features start to show.

the CM4-observatory data misfit increasingly reduced. At $n=7$, details of most of the magnetic features are distributed mostly in the southern part of the globe with northern part of the map mostly dominated by zero magnetic field intensity. Here the CM4 largely matches much of the observatory data in much of the globe, i.e. the model reproduces many of the features of the magnetic diurnal field map of the observatory station data. Due to the higher spherical harmonic truncation and regularisation, the diurnal variation field maps show a high level of detail while simultaneously suppressing small- scale noise. Figure 8 shows that the CM4 did a good job in predicting the observatory station data, as the difference between the model and data is very small at all the hours of the day. To a large extent the CM4 gives a good description of the features of the diurnal variation field away from quiet time all over the globe at spherical harmonic degree $7(n=7)$.

Figures 5-8 show that as we move to higher spherical harmonic degree truncation, the CM4 prediction of the observatory station data appears qualitatively better. This is seen in the model-data misfit, which reduces considerably. How- 
CM4

00 UT

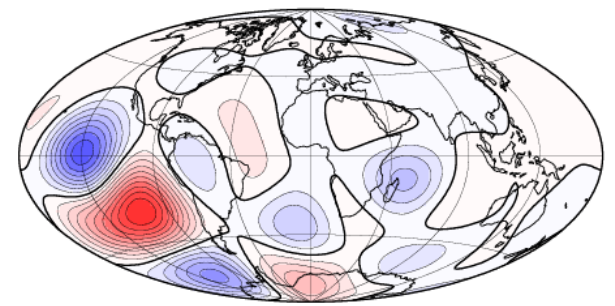

$-0.228 \mathrm{E}+03$ W $+0.228 \mathrm{E}+03$

06 UT

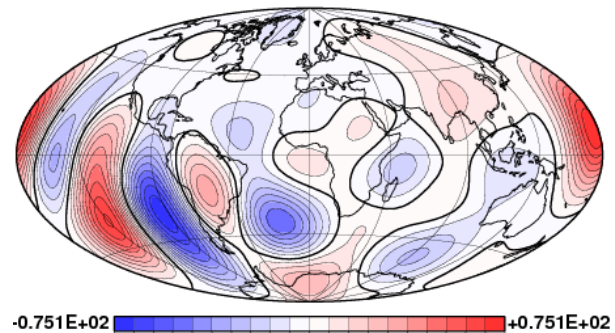

12 UT

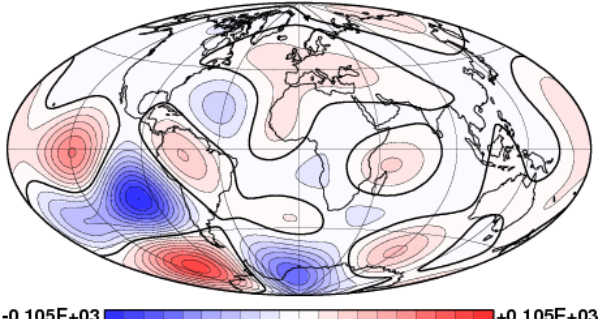

$-0.105 \mathrm{E}+03 \square+0.105 \mathrm{E}+03$

\section{UT}

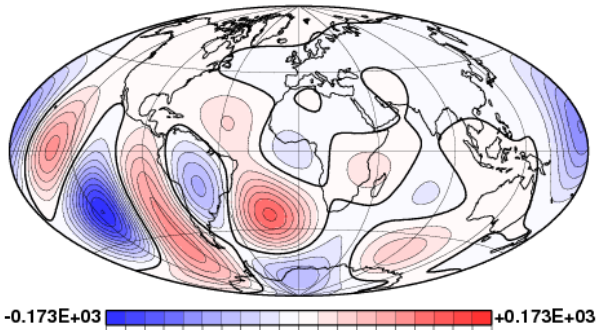

OBS DATA

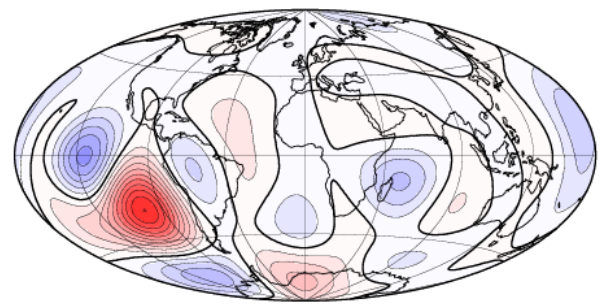

$- 0 . 1 3 5 \mathrm { E } + 0 4 \longdiv { + } + 0 . 1 3 5 \mathrm { E } + 0 4$
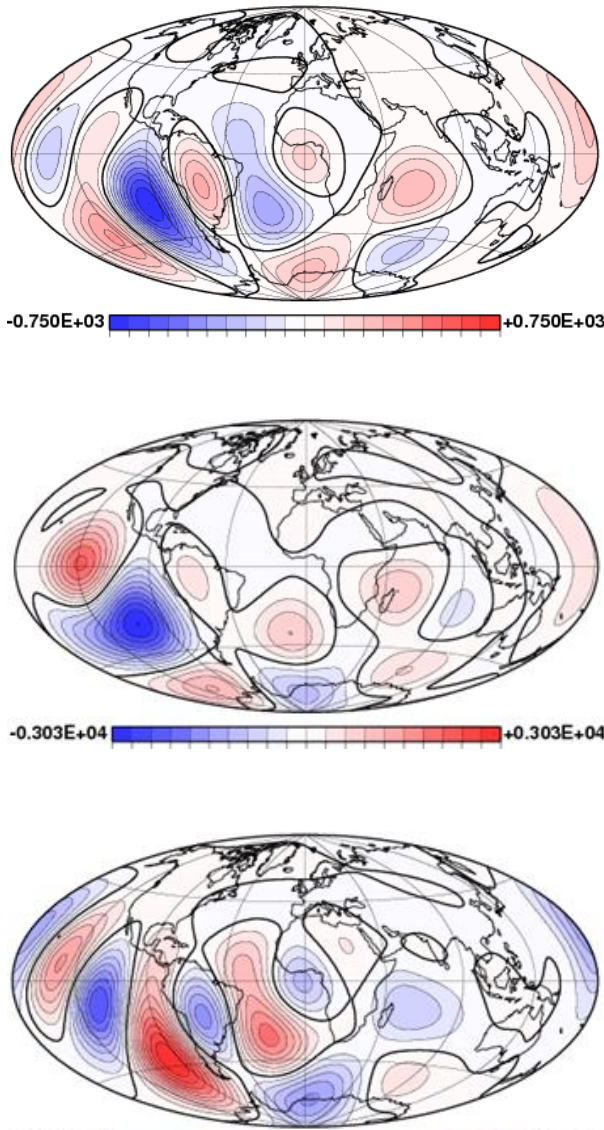

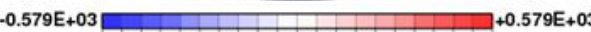

Figure 8. Similar to Fig. 5 but for spherical harmonic degree truncation $7(n=7)$. Better and improved coherence between the CM4 and observatory station data, i.e. better prediction of the observatory station data by the CM4.

ever, this relationship between increasing spherical harmonic degree truncation and reduction in misfit can be restricted by the data quality, quantity, coverage or spread. We also observed that as we increase the spherical harmonic degree truncation, the model-data roughness increases (Fig. 9), as small-scale features become capable of being incorporated. This does little to improve the complexity of the field or change the configuration, which is seen when increasing the spherical harmonic degree truncation to degree 8 or higher (not shown). This increase in the spherical harmonic degree truncation, reduction in model-data misfit and increase in roughness have a toll on the model-data viability as seen in the high-intensity values at higher spherical harmonic degree truncations. At higher spherical harmonic degree truncation (i.e. $n>5$ ), while the model prediction of the data is qualitatively better, it starts to go horribly wrong quantitatively as the roughness also increases, thus risking spurious features where there are no data to constrain the model. This is be- 


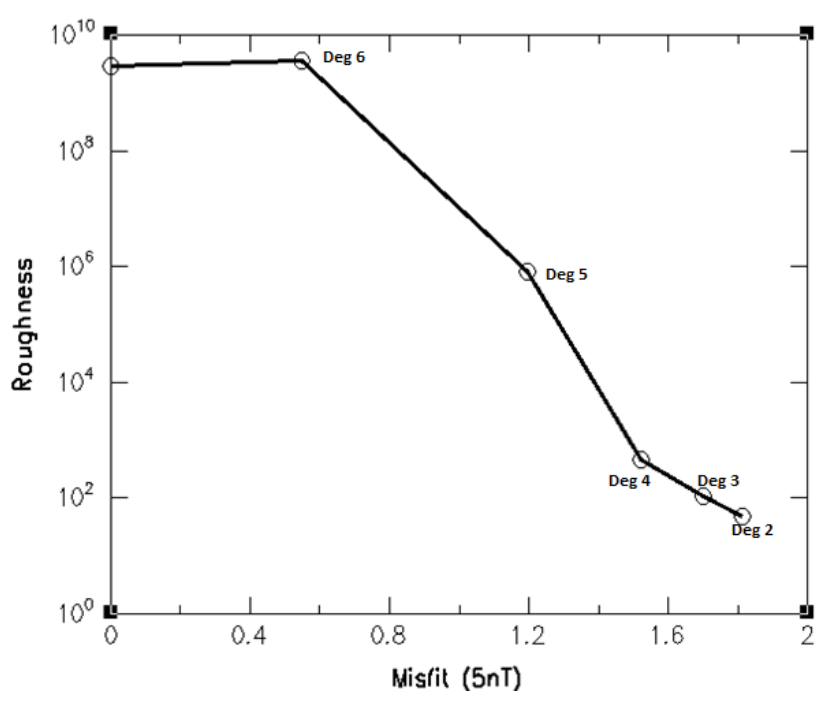

Figure 9. Plot of roughness against misfit of spherical harmonic degree truncation ( $n=2,3,4,5$ and 6). The roughness gives you a measure of when the data-model misfit starts to go wrong, which is at $n>5$.

cause, by including terms greater than degree 5 , there are too many free parameters that are unaccounted for. Here, even though the misfit between the model and data is considerably reduced, the roughness increases dramatically. This is illustrated graphically in Fig. 9.

\section{Conclusions}

Using global distribution of magnetic observatory station data, we study and analyse the geomagnetic diurnal variation field for days away from quiet time or moderately disturbed time. The main objective of the study is to look at the CM4 predictability of ground variation of the geomagnetic diurnal field for days away from quiet time. The main findings in the study can be summarised as follows.

The extension we applied to the CM4 modelling code by updating the data for the external field parts of the geomagnetic field appears to be working. This showed in the plots of the CM4 and observatory station data comparison in Figs. 3 and 4 . The various plots show features for different geographical locations which are in agreement with the diurnal variation for the different locations.

For the diurnal variation for days away from quiet time, the CM4 produces good prediction by matching the regional type features of the observatory station data reasonably well in all three geomagnetic components (XYZ). But the model did not match the observatory station data during the shorttime-period features where we observe rapid variations and fluctuations. This is hardly surprising as the CM4 can probably model periods down to $6 \mathrm{~h}$. These short-term periods are where we observe the largest misfit between the CM4 and the observatory station data in the plots.

Using relative rms misfit values as a tool for comparison between the CM4 predictions and the observatory station data for both quiet-time and non-quiet-time periods, and also within and outside the timespan of the CM4, we observed values less than 1 for most of the locations. One is the threshold for when the model explains precisely none of the data. This is a quantitative means of comparison against the qualitative means shown in our plots. These misfit values justify the extension of the CM4 outside the period the original model dataset covers, and confirmed its workability outside this period. However, its less clear that it works well for disturbed time, especially for regions like Oceania and Asia where we observe relative misfits very close to 1 for some components.

The global maps of the $\mathrm{Z}$ component of the geomagnetic diurnal variation field at the Earth's surface truncated at different spherical harmonic degrees and for different universaltime hours show that as we move to higher degree truncations, the CM4 predictions of the observatory station data appear better qualitatively. The model-data misfit is reduced, but the roughness is increased as seen in the high-intensity values of several hundreds of nanoteslas (the external field components should be a few tens of nanoteslas).

In closing, for the geomagnetic diurnal variation field for days away from quiet time, the CM4 performed reasonably well during these particular periods under study, giving a good description of the field, despite the lack of active data in the original model dataset. The model prediction is much better than expected. The point at which we usually say the model stops producing useful data is when the magnetic indices exceed the values for quietness put into the original dataset. If we say that the model appears to work inside the area of supposed dysfunction, are we being too strict with our limits on when the model can produce useful predictions? There appears to be some scope for inputting active datasets into the model to broaden its functions. Perhaps, we could further improve the performance of CM4 during active time or away from quiet time. However, further work still needs to be done to say how active that data can be while still acting to improve the model.

\section{Data availability}

The research data used in this study were taken from the INTERMAGNET website. The data are free and publicly available. They are definitive geomagnetic ground observatory data recorded from available worldwide locations for periods between May and September 2006. They can be accessed via http://www.intermagnet.org. 
Acknowledgements. E. Onovughe wishes to thank the Tertiary Education Trust Fund (TETFUND), Nigeria, for sponsorship of his $\mathrm{PhD}$ programme at the University of Liverpool. Appreciation also goes to Richard Holme (PhD supervisor), whose advice, direction and guidance was instrumental to most of the results of this study. Also, the author would like to thank the staff of the geomagnetic observatories and the INTERMAGNET programme for supplying the dataset used in carrying out this study. The analysis of this study was carried out as part of the author's postgraduate research study at the University of Liverpool. Finally, the author sincerely acknowledges the anonymous reviewers, whose comments and suggestions greatly contributed to improving the final manuscript as published.

The topical editor, S. Milan, thanks C. Nabert and one anonymous referee for help in evaluating this paper.

\section{References}

Campbell, W. H.: Annual and semi-annual changes of the quiet daily variations $(\mathrm{Sq})$ in the geomagnetic field at North America locations, J. Geophys. Res., 87, 785-796, 1982.

Campbell, W. H.: An Introduction to Quiet Daily Geomagnetic Fields, Pure Appl. Geophys., 131, 315-331, 1989.

Campbell, W. H.: Introduction to geomagnetic fields, Vol. 1, Cambridge University Press, 290 pp., 1997.

Chen, G., Xu, W., Du, A., Wu, Y., Chen, B., and Liu, X.: Statistical characteristics of the day-to-day variability in the geomagnetic Sq field, J. Geophys. Res., 112, A06320, doi:10.1029/2006JA012059, 2007.

Courtillot, V. and Le Mouel, J.-L.: Time variations of the Earth's magnetic field: from daily to secular, Annu. Rev. Earth Pl. Sc., 16, 389-476, 1988.

ESA SY-001 (2): Swarm: The Earth's magnetic field and environment explorers, ESA Publications Division, ESTEC, Noordwijk, 2005.

Gonzalez, W. D., Joselyn, A., Kamide, Y., Kroehl, H. W., Rostoker, G., Tsurutani, B. T., and Vasyliunas, V. M.: What is a geomagnetic storm?, J. Geophys. Res., 99, 5771-5792, 1994.

Graham, G. and Watchmaker, F. R. S.: An account of observations made of the variation of the horizontal needle at London, in the latter part of the year 1722, and beginning of 1723, Phil. Trans., 33, 96-107, 1724.

Hamid, N. S. A., Liu, H., Uozumi, T., Yumoto, K., Veenadhari, B., Yoshikawa, A., and Sanchez, J. A.: Relationship between the equatorial electrojet and global $\mathrm{Sq}$ currents at the dip equator region, Earth Planet. Space, 66, 146, doi:10.1186/s40623-0140146-2, 2014.

Hamilton, B.: Modelling the quiet-time geomagnetic daily variations using observatory data, GEOSPACE Consortium Meeting, Edinburgh, UK, 5-9 January, 2009.

Hamilton, B., Ridley, V. A., Beggan, C. D., and Macmillan, S.: The BGS magnetic field candidate models for the 12th generation IGRF, Earth Planet. Space, 67, 69, doi:10.1186/s40623-0150227-x, 2015

Hibberd, F. H.: The geomagnetic sq variation-annual, semi-annual and solar cycle variations and ring current effects, J. Atmos. Sol.Terr. Phy., 47, 341-352, 1985.
Hitchman, A. P., Lilley, F. E. M., and Campbell, W. H.: The quiet daily variation in the total magnetic field: global curves, Geophys. Res. Lett., 25, 2007-2010, 1998.

Joselyn, J. A.: Geomagnetic quiet day selection, Pure Appl. Geophys., 131, 333-341, 1989.

Kane, R. P.: Geomagnetic Field Variations, Space Sci. Rev., 18, 413-540, 1976.

Klausner, V., Papa, A. R. R., Mendes, O., Domingues, M. O., and Frick, P.: Characteristics of solar diurnal variations: A case study based on records from the ground magnetic station at Vassouras, Brazil, J. Atmos. Sol.-Terr. Phy., 92, 124-136, 2013.

Kobea, A. T., Amory-Mazaudier, C., Do, J. M., Lühr, H., Houngninou, Vassal, J., Blanc, E., and Curto, J. J.: Equatorial electrojet as part of the global circuit: a case-study from the IEEY, Ann. Geophys., 16, 698-710, doi:10.1007/s00585-998-0698-1, 1998.

Kono, M.: Geomagnetism in perspective, in: Treatise in Geophysics, edited by: Schubert, G., 5, 1-31, 2007.

Langel, R. A., Sabaka, T. J., Baldwin, R. T., and Conrad, J. A.: The near-Earth magnetic field from magnetospheric and quiet-day ionospheric sources and how it is modelled, Phys. Earth Planet In., 98, 235-267, 1996.

Le Sager, P. and Huang, T. S.: Ionospheric currents and fieldaligned currents generated by dynamo action in an asymmetric earth magnetic field, J. Geophys. Res., 107, 1025, doi:10.1029/2001JA000211, 2002.

Lester, M., Milan, S. E., Provan, G., and Wild, J. A.: Review of ionospheric effects of solar wind magnetosphere coupling in the context of the expanding contracting polar cap boundary model, Space Sci. Rev., 124, 117-130, 2006.

Lilley, F. E. M., Hitchman, A. P., and Wang, L. J.: Time-varying effects in magnetic mapping: amphidrones, doldrums and induction hazard, Geophysics, 64, 1720-1729, 1999.

Mandea, M. and Purucker, M.: Observing, modelling and interpreting magnetic fields of the solid Earth, Surv. Geophys., 26, 415459, 2005.

Mandea, M., Luhr, H., Korte, M., Balasis, G., Linthe, H.-J., Hemant, K., Pulz, E., Ritter, P., Rother, M., Stolle, C., Thebault, E., and Wardinski, I.: A comprehensive view of the Earth's magnetic field from ground and space observations, Zweijahresbericht GeoForschungsZentrum Potsdam 2004/2005, 63-76, 2006.

Matsushita, S. and Maeda, H.: On the geomagnetic solar quiet daily variations during International Geophysical Year, J. Geophys. Res., 70, 2535-2558, 1965.

Maus, S., Rother, M., Hemant, K., Stolle, C., Lühr, H., Kuvshinov, A., and Olsen, N.: Earth's lithospheric magnetic field determined to spherical harmonic degree 90 from CHAMP satellite measurements, Geophys. J. Int., 164, 319-330, 2006.

Menvielle, M. and Marchaudon, A.: Geomagnetic indices in solarterrestrial physics and space weather, in: Space weather: Research towards applications in Europe, Astrophysics and space science library, Springer, Dordrecht, Neth, 344, 277-288, 2007.

O'Brien, T. P. and McPherron, R. L.: Forecasting the ring current index $D_{\text {st }}$ in real time, J. Atmos. Sol.-Terr. Phy., 62, 1295-1299, 2000.

Olsen, N. and Mandea, M.: Rapidly changing flows in the Earth's core, Nat. Geosci., 1, 390-394, 2008.

Olsen, N., Sabaka, T. J., Mandea, M., Rother, M., Toffner-Clausen, L., and Choi, S.: CHAOS - A model of Earth's magnetic field de- 
rived from CHAMP, Ørsted, and SAC-C magnetic satellite data, Geophys. J. Int., 166, 67-75, 2006.

Olsen, N., Mandea, M., Sabaka, T. J., and Clausen, L. T.: CHAOS2 - A geomagnetic field model derived from one decade 10 of continuous satellite data, Geophys. J. Int., 179, 1477-1487, 2009.

Olsen, N., Mandea, M., Sabaka, T. J., and Toffner-Clausen, L.: The CHAOS-3 geomagnetic field model and candidates for the 11th generation IGRF, Earth Planet. Space, 62, 719-727, 2010a.

Olsen, N., Hulot, G., and Sabaka, T. J.: Sources of the geomagnetic field and the modern data that enable their investigation, in: Handbook of Geomathematics, edited by: Freeden, W., Nashed, M. Z., and Sonar, T., chap. 5, 106-124, 2010b.

Olsen, N., Luhr, H., Finlay, C. C., Sabaka, T. J., Michaelis, I., Rauberg, J., and Toffner-Clausen, L.: The CHAOS-4 15 geomagnetic field model, Geophys. J. Int., 197, 815-827, 2014.

Onovughe, E. and Holme, R.: The CM4 model prediction of ground variation of the geomagnetic diurnal field away from quiet time, Phys. Earth Planet In., 249, 1-10, 2015.

Potgieter, M. S.: Solar modulation of cosmic rays, Living Rev. Solar Phys., 10, 8-10, 2013.

Price, A. T.: Daily variations of the geomagnetic field, Space Sci. Rev., 9, 151-197, 1969.

Sabaka, T. J. and Baldwin, R. T.: Modeling the Sq magnetic field from POGO and MAGSAT satellite and cotemporaneous hourly observatory data: Phase 1, Technical Report Contract Report HSTX/G\&G9302, Hughes STX Corp., for NASA/GSFC Contract NAS5-31 760, NASA, 1993.

Sabaka, T. J. and Olsen, N.: Comprehensive modelling of the Earth's magnetic field: current status and future prospects, Proceedings of the 4th OIST conference, Copenhagen, Denmark, edited by: Stauning, P., 2003.

Sabaka, T. J., Olsen, N., and Langel, R. A.: A comprehensive model of the quiet-time near-Earth magnetic field: phase 3, Geophys. J. Int., 151, 32-68, 2002.

Sabaka, T. J., Olsen, N., and Purucker, M. E.: Extending Comprehensive models of the Earth's magnetic field with Oersted and CHAMP data, Geophys. J. Int., 159, 521-547, 2004.
Siscoe, G. and Odstrcil, D.: Ways in which ICME sheaths differ from magnetosheaths, J. Geophys. Res., 113, A00B07, doi:10.1029/2008JA013142, 2008.

Stening, R. J.: Longitude and seasonal variations of the sq current system, Radio Sci., 6, 133-137, 1971.

Stewart, B.: Hypothetical Views Regarding the Connection between the State of the Sun and Terrestrial Magnetism, Encyclopedia Britannica, 9th Edn., 16, 181-184, 1882.

Takeda, M.: The correlation between the variation in ionospheric conductivity and that of the geomagnetic Sq field, J. Atmos. Sol.Terr. Phy., 64, 1617-1621, 2002.

Thomson, A. W. P. and Lesur, V.: An improved geomagnetic data selection algorithm for global geomagnetic field modelling, Geophys. J. Int., 169, 951-963, 2007.

Tsyganenko, N. A.: A magnetospheric magnetic field model with a warped tail current sheet, Planet Space Sci., 37, 5-20, 1989.

Tsyganenko, N. A.: Modelling the Earth's magnetospheric magnetic field confined within a realistic magnetopause, J. Geophys. Res., 100, 5599-5612, 1995.

Tsyganenko, N. A.: Effects of the solar wind conditions on the global magnetospheric configuration as deduced from data-based field models, Proceedings of the 3rd International Conference on Substorms, Versailles, France, 12-17 May 1996, edited by: Rolfe, E. J. and Kaldeich, B., ESA European Space Agency, Paris, SP-389, p. 181, 1996.

Tsyganenko, N. A.: A model of the near magnetosphere with a dawn-dusk asymmetry-1, Mathematical structure, J. Geophys. Res., 107, 1179, doi:10.1029/2001JA000219, 2002.

Whaler, K. A.: Geomagnetism in the satellite era, Astron. Geophys., 48, 2.23-2.29, doi:10.1111/j.1468-4004.2007.48223.x, 2007.

Yizengaw, E., Moldwin, M. B., Zesta, E., Biouele, C. M., Damtie, B., Mebrahtu, A., Rabiu, B., Valladares, C. F., and Stoneback, R.: The longitudinal variability of equatorial electrojet and vertical drift velocity in the African and American sectors, Ann. Geophys., 32, 231-238, doi:10.5194/angeo-32-231-2014, 2014. 